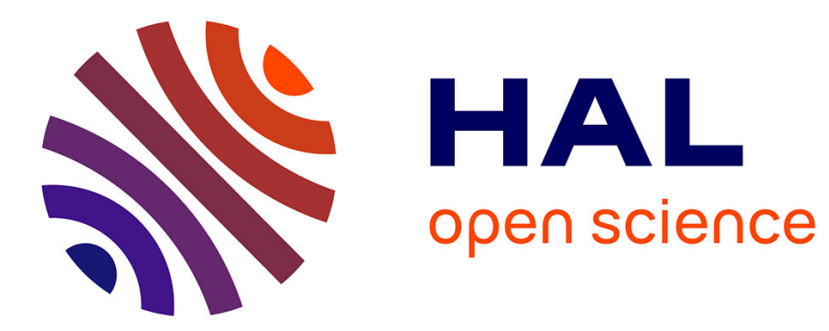

\title{
Nommer l'islam politique. Répertoire lexical d'un réformisme et ses réappropriations locales dans les noms de partis islamistes
}

Dilek Yankaya, Clément Steuer, Hassan Zouaoui

\section{- To cite this version:}

Dilek Yankaya, Clément Steuer, Hassan Zouaoui. Nommer l'islam politique. Répertoire lexical d'un réformisme et ses réappropriations locales dans les noms de partis islamistes. Mots: les langages du politique, 2019, 120, pp.145-163. 10.4000/mots.25394 . hal-02315715

\section{HAL Id: hal-02315715 \\ https://hal.science/hal-02315715}

Submitted on 22 Apr 2020

HAL is a multi-disciplinary open access archive for the deposit and dissemination of scientific research documents, whether they are published or not. The documents may come from teaching and research institutions in France or abroad, or from public or private research centers.
L'archive ouverte pluridisciplinaire HAL, est destinée au dépôt et à la diffusion de documents scientifiques de niveau recherche, publiés ou non, émanant des établissements d'enseignement et de recherche français ou étrangers, des laboratoires publics ou privés. 


\title{
Nommer l'islam politique. Répertoire lexical d’un réformisme et ses réappropriations locales dans les noms de partis islamistes
}

Article paru dans Mots. Les langages du politique, n 120, pp. 145-163.

https://dx.doi.org/10.4000/mots.25394

Dilek Yankaya

Sciences Po Aix

Clément Steuer

CNRS (Ladyss, UMR 7533)/ERC TARICA

Hassan Zouaoui

Université Ibn Zohr

\section{Financement}

Ce travail a reçu le soutien du programme de l'UE de recherche et d'innovation Horizon 2020 (programme TARICA, convention $n^{\circ}$ 695674) ; et de la région Rhône-Alpes (programme ExploraPro, 2011-2012).

\section{Résumé :}

Cet article interroge les modalités de nomination des partis islamistes autorisés à participer aux élections dans cinq pays (Maroc, Algérie, Tunisie, Égypte et Turquie). Tandis que ceux-ci sont perçus comme les représentants d'un conservatisme religieux, leurs noms puisent dans des répertoires lexicaux forgés par deux formes de réformisme, l’islamisme de la Nahda et le développementalisme nationaliste. Ces noms énoncent la quête d'un modèle de développement dont l'authenticité s'appuierait sur une double volonté de concrétiser des normes islamiques à l'échelle nationale et de répondre aux revendications des populations locales. L'article montre, néanmoins, comment, en l'absence d'un tel modèle authentique, quatre mots issus du répertoire lexical réformiste (justice, développement, liberté et renaissance) sont employés comme des «principes correcteurs ». Il contribue ainsi à expliquer comment le pragmatisme de l'islam politique se pratique selon un triptyque de logiques légaliste, d'affirmation électorale et de distinction politique.

\begin{abstract}
:
Based on a comparative study since the 1970s and in five countries (Morocco, Algeria, Tunisia, Egypt and Turkey), this article questions the methods of designation of Islamist parties. While these are perceived as representatives of religious conservatism, their names draw on lexical repertoires forged by two forms of reformism, Islamist of Nahda and nationalist developmentalist. Their names set out the quest for a development model whose authenticity would rely on a dual desire to implement Islamic norms at the national level and to respond to the demands of local populations. The article shows, however, how, in the absence of such an authentic model, four words from the reformist lexical repertoire (justice, development, freedom and renaissance) are used as "correcting principles". We thus
\end{abstract}


contribute to explain how the pragmatism of political Islam is practiced according to a three forms of logic: legalistic, electoral affirmation and political distinction.

\section{Resumen :}

Este artículo analiza cómo son construidos los de los partidos islámicos autorizados a participar en las elecciones en cinco países (Marruecos, Algeria, Túnez, Egipto y Turquía). Aunque los partidos islamistas son percibidos como representantes de un conservatismo religioso, sus nombres están asociados a repertorios léxicos construidos por dos corrientes reformistas, la islamista de la Nahda y la desarrollista nacionalista. Estos enuncian la búsqueda de un modelo de desarrollo cuya autenticidad se sustenta en una doble voluntad, la de concretizar normas islámicas a escala nacional, y la de responder a reivindicaciones de poblaciones locales. Sin embargo, el artículo muestra cómo, en ausencia de tal modelo auténtico, cuatro palabras del repertorio léxico reformista (justicia, desarrollo, libertad y renacimiento) son usadas como "principios correctores ». Contribuimos de esta forma a explicar cómo el pragmatismo del islam político se practica según un tríptico de lógicas, legalista, de afirmación electoral y de distinción política.

Mots clés:

Répertoire lexical, partis politiques, islam politique, circulations, Moyen-Orient et Afrique du Nord 


\title{
Nommer l'islam politique \\ Répertoire lexical d'un réformisme et ses réappropriations locales dans les noms de partis islamistes ${ }^{1}$
}

\author{
Dilek Yankaya, Clément Steuer, Hassan Zouaoui
}

La multiplication des partis islamistes et leurs récents succès électoraux en ont fait des acteurs incontournables des recompositions sociopolitiques en cours en Afrique du Nord et au Moyen-Orient. En Turquie, Adalet ve Kalkınma Partisi (Parti de la justice et du développement - AKP) se maintient au pouvoir depuis 2002. Son homonyme marocain, hizb al- 'adâla wal-tanmiyya (Parti de la justice et du développement - PJD) s'est placé en première position lors des élections de 2011. Ennahda (Parti de la renaissance) en Tunisie et hizb al-huriyya wal- `adâla (Parti de la liberté et de la justice) en Égypte ont remporté les premières élections législatives des «Printemps arabes ». En Algérie, plusieurs partis islamistes, d'inspiration frériste ou salafiste, dont Ennahda et le Front de la justice et du développement (jabhat al- `adâla wal-tanmiyya), occupent des sièges au parlement.

Traduisant un lexique islamique plus transversal que jamais (Bonnefoy et Burgat, 2018), leurs noms actuels présentent deux caractéristiques : absence de référence explicite à l'islam et usage fréquent de certains mots, comme justice, développement, renaissance (al-nahda, dont la prononciation dialectale est ennahda ou ennahdha). Pourquoi la référence à l'islam n'estelle pas explicite dans les noms de partis qui sont pourtant issus des mouvements islamistes ? Dans quels répertoires lexicaux leurs fondateurs ont-ils puisé ces mots pour exprimer leur engagement dans l'espace partisan ? Comment ces nominations ${ }^{2}$ sont-elles construites ?

Le constat de Paul Bacot (2010) sur la rareté des travaux consacrés à l’onomastique partisane est particulièrement vrai pour ce qui concerne les partis politiques d'Afrique du Nord et du Moyen-Orient. En effet, bien qu'une riche littérature ait démontré leur légitimité comme objet à part entière des sciences sociales (Baduel, 1996 ; Catusse, 2006a ; Beaumont et Guignard, 2016), seuls deux travaux portent sur leurs noms, qui sont d'ailleurs ceux de deux partis islamistes. Haoues Seniguer (2013) montre comment le PJD marocain poursuit une stratégie de sécularisation du vocabulaire pour maintenir une certaine représentation dicible de l'islam dans la course électorale. Ce constat s'applique également aux autres cas nationaux et s'exprime par l'abandon, quasi-généralisé, du répertoire lexical explicitement islamique depuis les années 1980. Clément Steuer (2013) étudie comment le nom du Hizb al-Wasat (Parti du centre) égyptien permet à ce parti de s'attribuer un positionnement central sur l'échiquier politique, tout en maintenant une référence implicite aux valeurs islamiques. Cette stratégie de nomination polysémique est également repérable dans la désignation d'autres partis politiques.

\footnotetext{
${ }^{1}$ Ce travail a reçu le soutien du Conseil européen de la recherche dans le cadre du programme de l'UE de recherche et d'innovation Horizon 2020, programme TARICA convention $n^{\circ} 695674$. Nous remercions chaleureusement Vincent Geisser, Belkacem Benzenine et Myriam Aït-Aoudia ainsi que nos évaluateurs pour leur relecture attentive.

${ }^{2}$ Nous employons ce terme pour désigner le processus, l’action de nommer (Siblot, 2001).
} 
Cet article soumet ces premiers éléments d'analyse à l'épreuve de l'étude comparatiste, qui est ici incontournable en raison de deux spécificités lexicales des partis islamistes : ils ont plusieurs fois changé de nom quelque soit le pays étudié, et certains mots circulent d'un pays à l'autre.

Cette étude porte sur cinq pays : Maroc, Algérie, Tunisie, Égypte ${ }^{3}$ et Turquie. Ce choix s'explique par l'interdépendance de longue date de ces sociétés politiques qui s’inscrivent, selon Jean-François Bayart (2016), dans un espace historique méditerranéo-moyen-oriental dont la cohérence renvoie à un moment d'historicité du passage de la domination impériale à la formation d'État-nations. Le «Printemps arabe » apparaît lui aussi comme un moment d'historicité commun, lorsque la Turquie faisait figure auprès des pays arabes de modèle à imiter, promettant stabilité démocratique et modernisation économique grâce à l'intégration officielle des mouvements islamistes dans la compétition politique, une " troisième voie » entre islam radical et autoritarisme séculier (Cook, 2017) ${ }^{4}$.

Notre analyse s'appuie principalement sur l'étude d'un corpus de noms de partis (voir tableau). Nous sollicitons également des sources secondaires et une série d'entretiens réalisés avec des responsables de partis. Dans les pays étudiés, le passage au multipartisme a été un processus contradictoire. Bien que visant à donner un cadre légal aux forces d'opposition, les lois sur les partis politiques posent des limites sémantiques aux choix lexicaux présidant à la nomination des partis. L'interdiction de créer un parti sur une base religieuse, un élément structurel commun aux cinq pays étudiés, explique sans doute la quasi-absence de référence explicite à l'islam, à la religion ou atà $A \underline{A}$ Dieu $^{5}$ dans les noms de partis autorisés à concourir aux élections ${ }^{6}$. Cette stratégie d'évitement d'un lexique ouvertement religieux est souvent pensée en rapport avec l'hypothèse du pragmatisme et de la modération des groupes islamistes qui, inclus dans la compétition électorale, se soumettent alors aux règles des régimes politiques pluralistes (Tazaghart, 2003 ; Amghar, 2008 ; Dris-Aït Hamadouche, 2009 ; Redissi, 2017). Ici, notre objectif est d'interroger se limitera à déerire lesles répertoires lexicaux qui alimentent les modalités dea nomination des partis islamistes.

Notre hypothèse est que les noms des partis islamistes en Afrique du Nord et au MoyenOrient traduisent l'expression de deux volontés politiques : celle d'énoncer un projet politique suivant une pensée islamique ressaisie comme universelle et intemporelle, et celle de façonner une mobilisation partisane viable dans les contextes politico-légaux nationaux. Ces deux ordres du politique, l'un transnational et l'autre national, correspondent à deux répertoires lexicaux, l'un forgé par l'histoire transnationale de l'islamisme et l'autre par la trajectoire

\footnotetext{
${ }^{3}$ Pour le cas égyptien, nous nous concentrerons sur la période d’ouverture politique de 2011-2013.

${ }^{4}$ Un dirigeant du parti Wasat égyptien nous avait d'ailleurs confirmé la proximité idéologique de son organisation avec l'AKP (Entretien, Le Caire, Avril 2008). Par ailleurs, la société de conseil en communication politique, présente derrière la stratégie de communication politico-électorale de l'AKP depuis sa formation en 2001, s'est portée volontaire pour conseiller les responsables d'Ennahda tunisien lors de sa campagne électorale en 2011 (entretien avec un responsable d'Ennahda tunisien, 25 avril 2018).

${ }^{5}$ À l'exception du Mouvement de la renaissance islamique en Algérie, et du Parti islamique du travail en Égypte, ce dernier étant d'ailleurs dénué de reconnaissance légale et a obtenu un seul député au Parlement de 2012, sur la liste d'une coalition électorale dominée par le PLJ.

${ }^{6}$ Maurice Tournier (2010) avait montré comment, dans un tout autre contexte puisqu'il s'agissait de la France de la Monarchie de Juillet, l'interdiction du désignant républicain par les lois de septembre 1835 avait permis l'essor du désignant radical en guise de substitut.
} 
nationale des États. La question du pragmatisme nous semble ouvrir ici une perspective d'analyse dans la mesure où ce mode d'action politique traduit l'engagement d'un dialogue multilatéral entre ce qui est pensé comme la raison universelle et impersonnelle - la religion et ce qui est perçu comme les multiples rationalités qui composent une société - le politique (Donegani, 2015). La construction des noms de partis islamistes, à chaque fois que ceux-ci ont changé de nom en raison d'une contrainte juridico-politique externe, d'une scission ou d'une reconfiguration internes, renvoie à un tel pragmatisme, repérable dans la manière dont les deux répertoires lexicaux sont sollicités dans une logique combinatoire à visées politicoélectorales.

Nous allons d'abord présenter l'historicité des répertoires lexicaux employés dans les noms des partis islamistes, ancrée dans le réformisme islamiste de la Nahda d'un côté et dans le développementalisme nationaliste de l'autre. Nous allons ensuite montrer comment ce répertoire transnational est retravaillé selon les configurations nationales. L'approche onomastique permettra ainsi d'aborder une nouvelle question, autre que celle classique de savoir si c'est la religion ou le politique qui va l'emporter lors de l'engagement partisan des mouvements islamistes (Kepel, 2000 ; Roy, 1992 ; Bayat, 1996 et 2013) : quelle empreinte l'entrée de l'islamisme dans l'espace partisan laisse-t-elle sur l'énonciation du politique ?

\section{Répertoire lexical réformiste : l’héritage commun d'une famille partisane hétérogène}

La politisation du rapport à l’islam se manifeste par une forte hétérogénéité partisane. S’il est ainsi possible de distinguer les mouvements dits salafistes, inspirés par la pensée wahhabite, de ceux appartenant au courant des Frères musulmans, héritiers du mouvement intellectuel réformiste de la Nahda, de nombreux processus d'emprunt et d'hybridation relient ces deux mouvances sur le plan empirique (Kepel, 2004 ; Lacroix, 2010) ${ }^{7}$. Les noms de partis politiques, qu’ils soient salafistes ou fréristes, présentent effectivement des éléments communs dans leurs noms et dans leur symbolisation, et renvoient à quatre principaux répertoires lexicaux. C’est d'abord le répertoire économique, avec l'usage fréquent de mots tels que développement, construction, réforme et travail. Le deuxième répertoire, religieux, emploie des mots comme renaissance, authenticité et lumière, qui ont des significations spécifiques, en lien avec le corpus religieux. Le troisième se réfère à une morale universelle, mobilisant des mots comme justice, liberté et paix. Le dernier répertoire renvoie à des désignations politico-spatiales, soit en lien avec l'espace politique, comme avec le mot centre, soit avec le territoire national, à travers le qualificatif national ou le nom du pays.

Les noms des partis politiques présentent deux modalités combinatoires de ces répertoires. La première modalité est l'adjonction de termes issus des différents répertoires lexicaux, et notamment des domaines économique et moral. C'est le cas du Parti de la justice et du développement en Turquie et au Maroc, du Front de la justice et du développement en Algérie. D’autres noms de partis, comme le Parti égyptien de la réforme et le Mouvement pour la réforme nationale algérien, combinent les répertoires économique et politico-spatial. La deuxième modalité de construction de noms de partis renvoie à l'usage de termes d'ordre

\footnotetext{
${ }^{7}$ La comparaison des stratégies politiques des organisations salafistes et fréristes est au-delà de l'objectif de cet article. Voir Algar (2002) et Rougier (2008). Pour la distinction entre « islamistes » et « néo-fondamentalistes » (Roy, 1992, 2002) ou entre réislamisation « par le haut » et « par le bas » (Kepel, 1991).
} 
moral ou explicitement islamique. C'est le cas d'Ennahda ${ }^{8}$ en Tunisie et du Mouvement de la renaissance islamique en Algérie, qui font directement référence à la Nahda, ainsi que du Mouvement de la Société pour la paix en Algérie, du parti Assala (« authenticité ») et du parti Nour (« lumière ») en Égypte.

Ces modalités de nomination, quoique différentes selon les contextes, ont néanmoins en commun d'exprimer, dans ses différents aspects, l'héritage réformiste foncièrement inscrit dans l'historicité des États au Moyen-Orient et en Afrique du Nord et qui trouve ses expressions politiques sous deux formes: comme catégorie d'action publique et comme répertoire d'action militante islamiste. Selon Béatrice Hibou (2005), les différentes formes de réformisme, républicain, colonial, nationaliste, avant et après les indépendances nationales ${ }^{9}$, sont des déclinaisons d'un même imaginaire réformiste ancré dans le réformisme ottoman. Bien que le contenu diffère chez les modernistes et les traditionnalistes, le réformisme fait consensus entre les gouvernants et les oppositions comme la seule modalité envisageable de l'exercice du pouvoir (Hibou, 2005 ; Catusse, 2006b). Le réformisme que revendiquent les mouvements islamistes contemporains et qui est l'objet de leur militantisme a été élaboré lors de différentes formes d'interaction avec l'Occident. D’abord la diffusion des idées des Lumières, et ensuite une prise de position critique vis-à-vis de celles-ci ont donné naissance à une pensée islamiste réformiste cherchant à se différencier du réformisme occidentaliste des élites dirigeantes : la Nahda. Ce premier réformisme consistait en un travail de révision de la foi, des pratiques et de la pensée islamiques (Bora, 2003). Les penseurs islamistes du $\mathrm{XX}^{\mathrm{e}}$ siècle ont gardé une orientation réformiste, mais dans un sens différent, cette fois-ci en vue de réformer la société pour forger une culture authentiquement islamique (Charif, 2006 ; Abbès et alii, 2014).

La Société des Frères musulmans (jamâ `at al-ikhwân al-muslimîn), dont le nom est raccourci en Frères musulmans (al-Ikhwân al-Muslimûn), fondée en 1928 en Égypte, est la première organisation inspirée de ce réformisme. Jamâ 'at en arabe - cemaat en turc - est un «nom propre collectif » possédant les propriétés sémantiques et référentielles décrites par Michelle Lecolle (2014) : une dénomination collective dont le référent est composé de personnes (des membres, des adhérents) regroupées selon un but commun ; ce groupe humain a un statut d'institution (au sens large). Cette dénomination exprime une volonté de concrétiser, à l'échelle locale l'Umma islamique, dans la mesure où jamâ 'a signifie d'abord le petit groupe de croyants qui font la prière (Abou Abdellah, nd). Il renvoie aussi, dans un sens plus large, à la communauté de croyants qui sont attachés au Coran et à la Sunna (paroles et actes du prophète Mohamed). Cet attachement constitue la raison d'être de cette communauté. Le mot prend ainsi un sens large désignant la communauté musulmane (Essayid, 2009). Jam'iyyat al-'ulamâ' al-muslimîn al-jazâ'iriyyîn, traduit par «l'Association des oulémas musulmans algériens», a été ensuite fondée en 1931 par des savants religieux se réclamant des mêmes sources intellectuelles que les Frères musulmans et prônant un islam réformé (Courreye, 2016).

\footnotetext{
${ }^{8}$ Prononciation dialectale de Al-Nahda, « la Renaissance ».

${ }^{9}$ La République arabe d’Égypte a été créée en 1922, la République de Turquie a été fondée en 1923, le Royaume du Maroc et la République tunisienne ont gagné leur indépendance en 1956, la République algérienne démocratique et populaire en 1962.
} 
Ce qui est pensé comme la résurgence de l'islamisme dans les années 1970 correspond à la multiplication de groupes organisés sous des noms propres collectifs renvoyant au même " cadre culturel, identitaire, affectif et mémoriel » (Paveau, 2008). C’est le cas de la chabîba islâmiyya (Jeunesse Islamique) fondée en 1969 au Maroc et de la jamâ ât islâmiyya en Tunisie dans les années 1970. Le cas turc présente une spécificité sémantique. Le mouvement islamiste a pris forme en 1975 sous le nom de Milli Görüş (Vision nationale). Le référentnom; görüş, signifie ${ }_{2}$ au sens propre, une vision atsens propre-et une opinion, une perspective, une manière de penser au sens figuré. L'usage du terme milli témoigne d'une inventivité

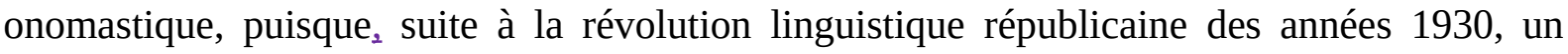
autre mot, ulus - d’origine mongole, donc non musulmane - lui a été préféré dans les discours et textes officiels, pour désigner la «nation» (Nisanyan, 2002). Signe de l'opposition à l’occidentalisation et à la laïcité, le langage utilisé par les acteurs islamistes turcs a pour spécificité lexicale de privilégier les mots d’origine arabo-persane dans leurs définitions prérépublicaines, inspirés selon eux du Coran, donc estimés plus adaptés à la culture « authentique », et aptes à réunir les communautés musulmanes (Strauss, 2008). L’usage du terme milli est aussi une stratégie de contournement de l'interdiction de l'usage de mots explicitement religieux, permettant ainsi de parler de la communauté musulmane sans parler d'islam.

La diffusion en langues nationales, à partir des années 1960, des ouvrages de penseurs islamistes a offert aux militants un nouveau vocabulaire politique de l'islam leur permettant d'aller au-delà des langages des politiques nationales (Kepel, 2000). Dans les années 1970, Rachid Ghannouchi, président d'Ennahda tunisien, a fait l'objet de critiques pour reprendre des mots «trop calqués sur les Frères musulmans » (Wolf, 2017). De leur côté, la plupart des membres fondateurs du PLJ ont été inspirés par la pensée des Frères musulmans ${ }^{10}$. « Qutb et Mawdudi ${ }^{11}$ m'ont ouvert l'horizon. Cela a été un tournant, j'ai commencé à m'intéresser à l'économie différemment ${ }^{12}$, disait un militant de l'AKP turc en parlant du début de son engagement islamiste. La circulation de cette littérature militante a ainsi provoqué une ardeur sémantique, participant à forger un « langage islamique » (Eickelman et Piscatori, 1996) dans la quête d'un modèle de développement, donnant lieu à une politique d'authenticité (asâla en arabe) structurée autour de ce qui relève du vrai islam (Mandaville, 2003). Le discours d'authenticité, loin de renvoyer à un retour en arrière, emploie les mots d'un renouvellement (Sayyid, 1997) orienté vers l'avenir: nahda, islâh, tanmiyya. Cette idéologie du développement authentique a ainsi formé un répertoire lexical itinérant ${ }^{13}$ à la disposition des militants et circulant d'un contexte politique à l'autre, d'une période à l'autre.

\footnotetext{
${ }^{10}$ Entretien avec un responsable du parti, Rabat, Maroc, Mars 2018.

11 Les ouvrages de l'écrivain Égyptien Sayyid Qutb apportent une forte critique du matérialisme, de la corruption et de la dégradation morale, et ceux du penseur pakistanais Abul Ala Mawdudi posent les jalons d'un modèle économique islamique (Chapra, 2004).

12 Entretien, Istanbul, Septembre 2007.

13 Nous nous inspirons du terme «théorie itinérante » (travelling theory), proposé par Edward Said pour désigner la circulation des idées (Said, 1984).
} 


\section{La Nahda et ses usages dans les contextes nationaux}

Nahda signifie renaissance, et réfère à l'énergie et à la puissance (Ibn Manzur, 2003 : 45604561). Le verbe de la même racine, nahada, veut dire "se relever ", " se remettre " ou « remettre debout ", avec une perspective active d' " être d'attaque ", d' " être prêt à » affronter. Ce mot est repérable dans le nom du parti islamiste tunisien et de l'un des principaux partis algériens, et aussi dans des programmes électoraux comme celui des Frères musulmans en 2012 nommé barnâmij al-Nahda, le programme de la Renaissance. La notion, elle, représente depuis le $19^{\mathrm{e}}$-XIX ${ }^{\mathrm{e}}$ siècle une position politique en faveur du changement et de la réforme. Présente dans le nom ou l'offre programmatique de plusieurs partis islamistes, elle attribue des significations spécifiques à ce réformisme vis-à-vis des enjeux politiques locaux.

Alors que le nom du Parti de l'authenticité (hizb al-asâla) constitue une référence explicite à la quête d'authenticité mentionnée supra, le nom du Parti égyptien de la réforme (hizb alislâh) réfère à tout ce qui est relatif à la réforme et à la lutte contre la corruption (Ibn Manzur, 2003 : 516). Le nom du Parti islamique du travail (hizb al- amal al-islâmi) porte également le sens de l'action pour le changement, car le verbe 'amala, riche de sens, se rapporte au travail autant pour soi-même que pour autrui (Ibn Manzur, 2003 : 3107-3108). Enfin, le nom du Parti de la construction et du développement (hizb al-binâ' wal-tanmiyya) met en avant le versant développementaliste, le terme al-binâ' eorrespondant-renvoyant ici à l'acte de «bâtir, construire, édifier $\Perp$, correspondant à un acte de mener au progrès et au développement (Ibn Manzur, 2003 : 94-95).

En Algérie, le Mouvement de la société islamique, parti créé en 1990, a pour base institutionnelle l'Association «Guidance et Réforme » (al-irshâd wal-islâh) (BoubaekeurBoubekeur, 2008). Par son slogan «l'islam, c'est la solution » il mettait le revivalisme islamique au centre de ses ambitions réformistes. En 1997, le parti a changé de nom pour devenir le Mouvement de la société pour la paix, tout en gardant son sigle ${ }^{14}$. Ce changement s'est accompagné de celui de son slogan, désormais devenu :-pouf « la paix, c’est la solution », qui représente moins un repositionnement moins-vis-à-vis du répertoire lexical quequ'une volonté de se démarquer nettement du par rapport attrecours atà un répertoire d'action violente. Les termes islâm, signifiant la "soumission à dieu », et salâm (« sauf », « intact », « en paix ») viennent de la même racine slm. Ils sont souvent pensés ensemble, l'islam étant revendiqué comme religion de paix (Greiner, 2018). Dans le contexte algérien d'alors, déchiré par la guerre civile, ce choix de nom vise ainsi à se différencier des mouvements islamistes qui menaient des actions violentes contre l’État. Le MSP, participant à la coalition gouvernementale de 1997 à 2012, s'est ainsi institué, pour certains, comme représentant les «islamistes de l’État ». De leur côté, les autres partis algériens présents au Parlement, le Mouvement de la renaissance islamique, le Mouvement pour la réforme nationale, issu d'une scission du précédent, et le Front de la justice et du développement portent des déclinaisons lexicales de la symbolique du progrès inscrite dans le répertoire de la Nahda.

\footnotetext{
${ }^{14}$ Identique à celui de la branche palestinienne des Frères musulmans, le Mouvement de la résistance islamique, Harakat al-muqâwama al-islâmiyya, ou Hamas, dont le nom renvoie à l'ardeur et à la détermination à défendre une cause (Ibn Manzur, 2003 : 994-995). Pour la stratégie de détournement du sigle
} 
Au Maroc, la Jeunesse islamique a été légalisée en 1988 sous le nom du Mouvement de la réforme et du renouveau (harakat al-islâh wal-tajdîd) - précédant la fondation du Parti de la justice et du développement en 1998 -, dont le nom se réfère explicitement à la pensée réformiste de la Nahda (Dialmy, 2009). Le choix d'une lampe à huile (misbah) comme emblème du PJD s’inscrit également dans ce répertoire symbolisant «la renaissance économique et sociale du pays $»^{15}$. La lumière, nûr en arabe, a une puissance symbolique forte en islam et apparaît fréquemment dans le Coran pour signifier notamment le passage « des ténèbres à la lumière » (Bonnéric, 2012). Elle fait référence à la connaissance, à la foi et, dans le verset qui porte le même nom (âyat al-nûr, verset de la lumière), elle est assimilée à dieu (Berque, 1995). La notoriété de ce verset permet d'expliquer la mobilisation du symbole de la lumière sous différentes formes, textuelle dans le nom du Parti Nour égyptien et graphique dans les logos, comme la lampe à huile du PJD, l'ampoule de l'AKP et le soleil du Parti de la liberté et de la justice, ainsi que dans les emblèmes électoraux comme l'ampoule du Parti Wasat aux élections de 2011-2012 et la lanterne du Ramadan (fânûs) du Parti Nour. Cette symbolisation témoigne ainsi de la filiation de ces formations au répertoire lexical de la Nahda.

Quant au Parti de l'ordre national turc (Milli Nizam Partisi - MNP), celui-ci porte une revendication revivaliste dans sa charte :

Le MNP, qui n'est rien d'autre que la renaissance de l'essence tant matérielle que spirituelle [...], est fondé dans le but de transformer en actes les facultés morales et vertueuses (fazilet) se trouvant dans la nature de la nation... et d'apporter ainsi à notre communauté (millet) l’ordre (nizam), la sérénité, la justice (adalet) sociale, et à nos citoyens le bonheur (saadet) et le salut (selamet) $»^{16}(\mathrm{MNP}, 1970)$.

Cette charte, utilisant ces signifiants d'origine arabe, a fourni au mouvement « un vocabulaire extensif et haut en couleur » (Coakley, 1980) de la renaissance. Ce vocabulaire a assuré une continuité sémantique dans la succession des partis islamistes turcs, étant donné que c'est dans ces premières phrases qu'ont été puisés tous les noms de partis issus du mouvement de la Vision nationale ${ }^{17}$.

Enfin, en Tunisie, le choix, des dirigeants du Mouvement de la tendance islamique (MTI, harakat al-itijâh al-islami) de transformer, en 1988, l'intitulé de leur organisation en Ennahdha (hizb harakat al-Nahda - «parti du mouvement de la renaissance »), en se revendiquant explicitement de l'héritage réformiste musulman des $\mathrm{XIX}^{\mathrm{e}}$ et $\mathrm{XX}^{\mathrm{e}}$ siècles, représente selon son leader Rachid Ghannouchi :

Une seconde véritable naissance [...] non plus comme porte-parole d'islam [...] mais tout simplement comme un des partis politiques [qui ne propose] qu'une lecture de l’islam. » (cité dans Finianos, 2006).

\footnotetext{
${ }^{15}$ Entretien avec un responsable du parti, Settat, Maroc, Mars 2018.

${ }^{16}$ Charte de fondation du MNP, Nüve Matbaasi, Ankara, 1970.

${ }^{17}$ Le Parti du salut national (Milli Selamet Partisi) 1972-1981. Le Parti de la providence (Refah Partisi) 19831997. Le Parti de la vertu (Fazilet Partisi) 1997-2001. Le Parti de la félicité (Saadet Partisi) depuis 2001.
} 
Ce changement a traduit à la fois une volonté de rompre avec une longue période de répression du mouvement et de s’instituer comme une force d'opposition légale, donc reconnue par le régime (Camau et Geisser, 2003). Certaines références à la Nahda sont cependant plus récurrentes que d'autres dans les noms de partis qui composent notre corpus.

\section{Parti ou mouvement ? Justice, développement et liberté. Les mots de la Nahda}

Un examen de ce dernier révèle d'abord une domination du " nom collectif classificatoire » (Lecolle, 2014) parti (hizb en arabe, parti en turc), utilisé par l'ensemble des organisations partisanes turques, marocaines et égyptiennes. Cette exclusivité de la catégorie parti - « signe dominant du politique » (Tournier, 2010 : 31) - dans les noms de ces trois pays tranche avec la situation algérienne, où l'on trouve trois organisations islamistes se désignant comme mouvements (haraka), l'une comme front (jabha), et pas une seule comme parti. À l'origine, le terme hizb désigne un groupe de partisans (Ibn Manzur, 2003 : 309), alors que haraka réfère étymologiquement à une force motrice qui contribue à la mise en mouvement d'une chose (Ibn Manzur, 2003 : 844-849) et jabha renvoie à la fois à l'action de prier (lorsque le croyant pose son front sur le sol), à celle de diriger (jabhat al-qawm, le chef d'un groupe) et à un groupe de personnes (Ibn Manzur, 2003 : 540-541). Le parti islamiste tunisien emploie de son côté le substantif mouvement (haraka): son nom officiel complet est celui de hizb harakat al-Nahda ("parti du mouvement de la Renaissance »). Ce nom révèle comment la nomination d'un parti politique est un processus conflictuel au croisement de deux modalités d'action politique défendues par les militants. Les contraintes de ce dernier expliquent le changement de nom du Mouvement de tendance islamique pour Ennahda. Toutefois, le substantif haraka est un élément de compromis fait par les défenseurs d'une orientation "partisane » (hizbiste) de conquête du pouvoir aux tenants d'une orientation "mouvementiste » (harakiste) prônant la réforme de la société par le bas en retour d'une participation au champ politique légal (Camau et Geisser, 2003).

Ces noms collectifs classificatoires se voient adjoints dans la grande majorité des cas un ou deux compléments du nom, parmi lesquels les mots justice et développement, parfois liberté occupent une place de choix. Lorsque ceux-ci renvoient au répertoire de la Nahda, ils sont investis de représentations politiques foncièrement ancrées dans les trajectoires nationales, et dans les revendications que les fondateurs des partis supposent être celles des populations locales. Le parti islamiste tunisien Ennahda utilise ainsi ces mots pour décrire son objectif : « une Tunisie de la liberté, de la justice et du développement [...] tout en ayant comme points de repère les valeurs islamiques et les acquis de notre expérience tunisienne ${ }^{18}$. De son côté, le programme électoral du parti des Frères musulmans égyptiens créé en 2011, le Parti de la liberté et de la justice, renvoie à ce que ses fondateurs considèrent comme

Les plus importants objectifs de la charî`a. Les libertés fondent la justice ; et avec la justice vient le développement authentique ; et de là l’Égypte s'engage sur la voix du leadership à la hauteur de son histoire ${ }^{19}$.

\footnotetext{
${ }^{18}$ Programme du Mouvement Ennahdha, 2011.

${ }^{19}$ https://egyptianpartiesprograms.wordpress.com/.
} 
C'est aussi une telle démarche d'inscription locale qui caractérise les propos du leader du Front de la justice et du développement est-créé en 2011 en Algérie : "Après une étude objective, nous avons estimé que la justice et le développement sont les plus grands défis de l'Algérie $»^{20}$, explique-t-il pour écarter l'hypothèse de l'importation du label de son parti à partir de l'AKP turc.

Ce sont pourtant les militants islamistes marocains qui sont à l'initiative de ce couple lexical, dans la mesure où la création du Parti de la justice et du développement (PJD) en 1998 précède celle de l’AKP, fondé en 2001. La loi relative aux partis politiques interdit la fondation d'un parti politique sur des bases religieuses (Loi $n^{\circ} 29-11$ ) dans un État se revendiquant comme un «État musulman» selon la Constitution, et ainsi constitue un dispositif d'évitement de tout risque d'émergence d'un gouvernement alternatif mobilisant l'islam. Ce gouvernement du religieux est bien compris, à la manière des fondateurs d'Ennahda tunisien, par ceux du PJD, qui avancent l'argument des configurations politiques nationales dans leur modalité de nommer leur parti : «La religion devrait être considérée comme la raison d'être de la monarchie [...] et le PJD ne peut pas adopter le modèle de l'AKP, mais il souhaite voir émerger à l'avenir un modèle marocain ${ }^{21}$. Un membre fondateur a expliqué comment le choix du nom du parti relève d'une négociation entre le répertoire lexical religieux et les limites sémantiques constitutionnelles :

Ce nom a été choisi car ces deux valeurs islamiques fondamentales font partie des principes constitutionnalisés [...] Nous voulons remédier aux faiblesses en matière de justice et développement à partir d'une référence islamique pour ainsi corriger les anomalies qui caractérisent la gestion des affaires publiques ${ }^{22}$.

Le mot justice ('adâla), signifiant «la justice divine», renvoie aussi à l'ensemble des procédures juridiques et politiques adoptées pour appliquer ou réaliser cette dernière (Ibn Manzur, 2003 : 430). En effet, selon le programme du PJD, la justice est mise en opération comme l'instauration des principes de la justice sociale, de la solidarité et l'accès des citoyens aux services publics et aux richesses. Le mot développement (tanmiyya), qui signifie " croissance », ou " augmentation » (ibid.: 341), prend, de son côté, un sens large qui considère l'homme comme étant l'objet d'un programme complet de développement spirituel, économique et social $^{23}$. La particularité de ce label consiste dans sa valeur en tant que ressource sémantique mobilisée dans la compétition politique, d'autant que le PJD cherche à se positionner sur l'échiquier politique comme un parti revendiquant « un réel projet de justice sociale, d'un enseignement fiable et d'une santé digne de ce nom, d'une fiscalité responsable et solidaire ", souligne un membre fondateur du $\mathrm{PJD}^{24}$. Le nom du parti est donc investi d'une double signification, à la fois économique et islamique, renvoyant à deux ordres de « l'énonciation du politique, matériel et moral » (Bayart, 1985).

\footnotetext{
${ }^{20}$ Interview d'Abdellah Djaballah, Algérie1.com, 10 mai 2012.

${ }^{21}$ Interview d'Abdelilah Benkirane, Al-Sharq al-Awsat, 27 novembre 2011.

22 Entretien avec un responsable du parti, Rabat, Maroc, Mars 2018.

${ }^{23}$ Site officiel du PJD : http://www.pjd.ma.

${ }^{24}$ Ibid.
} 
Le nom de l'AKP relève de la même démarche constructionnelle selon l'explication de l'ancien ministre de la Culture du Parti de la Providence (1996-1997), qui dit avoir proposé ce nom à Recep Tayyip Erdoğan en 2001 :

Je lui ai dit que ce nom portait une idée d'action, de nouveau départ et qu'il donnait [comme sigle] AK Parti. AK représente la blancheur, la pureté. Car il fallait du développement, mais il nous fallait aussi de la spiritualité, c’est-à-dire la justice ${ }^{25}$

Le nom du parti appelle alors à une entrée dans la modernité économique avec quelques corrections d'ordre moral sur la spiritualité, entendue comme remède aux maux de l'esprit égoïste et de la vie matérialiste (Ünsaldi, 2010). L'appel à " l'action », à " un nouveau départ ", s’inscrit dans l'univers de sens de la Nahda et est également symbolisé par le sigle AK Parti (littéralement : " le parti blanc »). Le parti s'est ainsi réapproprié la symbolique attribuée à la couleur blanche, perçue comme celle de l'hygiène mais aussi de l'innocence, de la pureté, sans oublier son évocation de la lumière divine (Pastourau et Simonnet, 2007) : «le Parti pur/propre ", proposé comme un discours avant même d'être pris dans le discours (Bacot, 2010) $)^{26}$.

\section{Énonciation réformiste du politique}

Considérés comme des forces politiques conservatrices, les partis islamistes mobilisent pourtant un répertoire lexical intimement réformiste. L'étude comparative des noms de partis islamistes dans le temps et l'espace montre comment la construction du répertoire de la Nahda ainsi que ses réappropriations sémantiques locales s'inscrivent dans les historicités d’États connectées par le transnationalisme de la pensée réformisme et l'impératif nationaliste du développementalisme. Partant de cette analyse, les formes partisanes de l'islamisme contemporain sont donc loin d'être des objets politiques importés. Elles sont les produits authentiques des trajectoires politico-juridiques des contextes nationaux dans lesquels elles évoluent aujourd'hui.

L’étude de Javier Albarracin et Paula Cusi (2012) sur les programmes électoraux, les discours et les décisions économiques adoptés par les partis islamistes montre cependant comment, même si le développement national était un grand thème de leur quête de pouvoir, c'est plutôt « une absence patente d'options de rupture ou d'alternatives révolutionnaires dans le domaine économique » qui caractérise leur action politique. En l'absence d'une véritable stratégie de développement authentiquement islamique, l'islam apparaît comme une "pratique discursive » (Addi, 2003) dans l'énonciation du politique par ces partis. Certains mots favoris de ce répertoire - renaissance, justice, développement et liberté - sont employés dans leurs noms, selon des modalités combinant différents types de répertoire lexical, « au sens de principes correcteurs » (Ünsaldi, $2010:$ 170).

\footnotetext{
${ }^{25}$ Milliyet, 22 mai 2012.

${ }^{26}$ Toutefois, bien que ce nom propre ait été officiellement établi, son usage n’a jamais été stabilisé dans la société. Le parti est appelé par deux noms AKP et AK Parti, le choix de désignation étant désormais un signe de positionnement par rapport au parti. Les militants et les sympathisants utilisent AK Parti tandis que les opposants disent AKP, selon la forme prototypique des sigles de partis turcs. Ce conflit d'appellation montre comment le nom propre collectif devient un enjeu de domination et d'opposition.
} 
Mobilisés par une stratégie de nomination polysémique, ces mots expriment trois dimensions de la mise en action partisane des mouvements islamistes : le souci légaliste d'intégrer la politique institutionnelle ; le souci d'affirmation électorale ${ }_{2}$ dans la mesure où ces mots sont mis à la disposition des citoyens en manque de repères et de valeurs morales dans ce monde « injuste » (Ünsaldi, ibid.) ; et la volonté de se démarquer à la fois d'autres partis politiques et d'autres catégories d'acteurs islamistes recourant à des répertoires d'actions violents (Brésillon et Ayari, 2018). Concrètement, c'est cela le pragmatisme des islamistes : leur volonté de retravailler les frontières supposées séparer le religieux du non religieux par des processus de recadrage des revendications locales dans un vocabulaire islamique retravaillé selon les contextes nationaux. 


\begin{tabular}{|c|c|c|c|c|c|}
\hline Pays & $\begin{array}{l}\text { Nom du parti en } \\
\text { français }\end{array}$ & $\begin{array}{l}\text { Nom du parti en } \\
\text { langue nationale }\end{array}$ & Transcription & $\begin{array}{l}\text { Année de } \\
\text { fondation }\end{array}$ & Statut \\
\hline \multirow[t]{2}{*}{ Turquie } & $\begin{array}{l}\text { Parti de la justice } \\
\text { et du développe- } \\
\text { ment }\end{array}$ & $\begin{array}{r}\text { Adalet ve Kal- } \\
\text { kinmaPartisi }\end{array}$ & $\begin{array}{l}\text { Adalet ve Kalkın- } \\
\text { ma Partisi }\end{array}$ & 2001 & Gouvernement \\
\hline & Parti de la félicité & Saadet Partisi & Saadet Partisi & 2001 & $\begin{array}{l}\text { Opposition } \\
\text { hors parlement }\end{array}$ \\
\hline Tunisie & Ennahdha & النهضة & $\begin{array}{l}\text { ennahdha - hara- } \\
\text { kat al-nahḍa }\end{array}$ & 1989 & Gouvernement \\
\hline Maroc & $\begin{array}{l}\text { Parti de la justice } \\
\text { et du développe- } \\
\text { ment }\end{array}$ & |حزب التدالة & $\begin{array}{l}\text { hizb al-`adâla wal- } \\
\text { tanmiyya }\end{array}$ & 1998 & Gouvernement \\
\hline \multirow[t]{7}{*}{ Égypte } & $\begin{array}{l}\text { Parti de la liberté } \\
\text { et de la justice }\end{array}$ & حزب الحرية والعدالة & $\begin{array}{l}\text { hizb al-huriyya } \\
\text { wal-`adâla }\end{array}$ & 2011 & $\begin{array}{l}\text { Interdit en } \\
2013\end{array}$ \\
\hline & $\begin{array}{l}\text { Parti de la lu- } \\
\text { mière - Parti } \\
\text { Nour }\end{array}$ & حزب النور & hizb al-nûr & 2011 & $\begin{array}{l}\text { Présen } \\
\text { t au parlement }\end{array}$ \\
\hline & $\begin{array}{l}\text { Parti construction } \\
\text { et développement }\end{array}$ & حزب البناء والتنمية & $\begin{array}{l}\text { hizb al-binâ' wal- } \\
\text { tanmiyya }\end{array}$ & 2011 & $\begin{array}{l}\text { Exclu du jeu } \\
\text { politique - Vi- } \\
\text { sé par des pro- } \\
\text { cédures d'in- } \\
\text { terdiction }\end{array}$ \\
\hline & $\begin{array}{l}\text { Parti de l'authen- } \\
\text { ticité - Parti As- } \\
\text { sala }\end{array}$ & حزب الأصالة & hizb al-asâla & 2011 & $\begin{array}{l}\text { Exclu du jeu } \\
\text { politique }\end{array}$ \\
\hline & $\begin{array}{l}\text { Parti du centre - } \\
\text { Parti Wasat }\end{array}$ & حزب الوسط & hizb al-wasat & 1996 & $\begin{array}{l}\text { Autorisé en } \\
2011 \text { - Hors } \\
\text { parlement }\end{array}$ \\
\hline & $\begin{array}{l}\text { Parti islamique } \\
\text { du travail }\end{array}$ & الاسختمي العمل & $\begin{array}{l}\text { hizb al-`amal al- } \\
\text { islâmi }\end{array}$ & 1978 & $\begin{array}{l}\text { Interdit en } \\
2000\end{array}$ \\
\hline & $\begin{array}{l}\text { Parti égyptien de } \\
\text { la réforme }\end{array}$ & حزب الإصلاح & hizb al-islâh & 2011 & Hors parlement \\
\hline \multirow[t]{3}{*}{ Algérie } & $\begin{array}{l}\text { Mouvement de la } \\
\text { société pour la } \\
\text { paix }\end{array}$ & - حركة دجتمع السلم & $\begin{array}{l}\text { harakat mujtama` } \\
\text { al-salam - hamas }\end{array}$ & $\begin{array}{l}1990 \\
(1997 \\
\text { sous son } \\
\text { nom } \\
\text { actuel })\end{array}$ & Parlement \\
\hline & $\begin{array}{l}\text { Mouvement de la } \\
\text { renaissance isla- } \\
\text { mique }\end{array}$ & الإسلامية - النهضة & $\begin{array}{l}\text { harakat al-nahda } \\
\text { al-islâmiyya- En- } \\
\text { nahda }\end{array}$ & 1989 & Parlement \\
\hline & $\begin{array}{l}\text { Front de la jus- } \\
\text { tice et du déve- } \\
\text { loppement }\end{array}$ & جبهة العدالة والتنمية & $\begin{array}{l}\text { jabhat al-`adâla } \\
\text { wal-tanmiyya }\end{array}$ & 2011 & Parlement \\
\hline
\end{tabular}




\begin{tabular}{|l|l|r|l|l|l|}
\hline $\begin{array}{l}\text { Mouvement pour } \\
\text { la réforme natio- } \\
\text { nale }\end{array}$ & $\begin{array}{r}\text { harakat al-islâh al- } \\
\text { watani }\end{array}$ & 1999 & Parlement \\
\hline
\end{tabular}

\section{Références}

ABBÈs Makram, Mongin Olivier, TENEZAKIS Xenophon, 2014, «L'art de gouverner en Islam », Esprit, n 8 , p. 163-186.

Abou AbDellah Mohamed ben Abdellah Al-khorchi al Maliki, nd., Charhe Mokhtassare khalili li- al Kkochi, Beyrouth, Éditions dar-Al Fikr.

ADDI Lahouari, 2003, «Pluralisme politique et islam dans le monde arabe », Pouvoirs, $\mathrm{n}^{\circ} 1$, p. 85-95.

AlbarRacin Javier, CUSI, Paula, 2012, «Les islamistes : analyse d'un agenda économique en construction », Confluences Méditerranee n 4, p. 119-134.

Algar Hamid, 2002, Wahhabism: A Critical Essay, New-York, Islamic Publications International.

AMGHAR Samir, 2008, « Les partis islamistes en Algérie : structures révolutionnaires ou partis de gouvernement ? », Maghreb-Machrek, n¹94, p. 529-541.

BAcot Paul, 2010, " Développement et diversification d'une onomastique politique », Mots. Les langages du politique, $\mathrm{n}^{\circ}$ 94, novembre, p. 47-56.

BADUEL Pierre-Robert, (éd.), 1996, «Les partis politiques dans les pays arabes-1. Le Machrek », Revue des Mondes Musulmans et de la Méditerranée, n 81-82, p. 9-51.

BAYART Jean-François, 2016, «Dessine-moi un MENA !”, ou l'impossible définition des “aires culturelles” », Sociétés politiques comparées, vol. 38, p. 1-28.

- 1985 « L’énonciation du politique », Revue française de science politique, p. 343-373.

BAyAT Asaf, 1996, « The Coming of a Post-Islamist Society », Critique, p. 43-52.

- (éd.), 2013, Post-islamism: The Changing Faces of Political Islam, Oxford and New-York, Oxford University Press.

BeAumont Robin, Guignard Xavier, 2016, «Partis et recompositions politiques : quelques enseignements du « Printemps arabe ». Confluences Méditerranée, no. 3, p. 9-19.

BeChIR-AyARI Michaël, Bresillon Thierry, 2018, « Le "retour" du parti Ennahda sur la scène politique : de la normalisation démocratique au compromis autoritaire ? ", dans Tunisie. Une démocratisation au-dessus de tout soupçon Allal A. et Geisser V. éd., Paris, CNRS Éditions.

Berque Jacques, 1995, Le Coran. Essai de traduction, 1995 (édition revue et corrigée), Paris, Albin Michel. 
BonnEFoy Laurent, BuRgat François, 2018, « Dynamique et omniprésente diversité de la référence islamiste », Critique internationale, $\mathrm{n}^{\circ}$ 1, p. 11-19.

BONNÉRIC Julie, 2012, "Symboliser et figurer le divin en Islam classique : entre lumière naturelle et lumière artificielle », Journal Asiatique, vol. 300, n² 2, p. 761-776.

BORA Tanil, 2003, Türk Saginin Üç Hali [Les trois états de la droite turque], Istanbul, Birikim.

BoubeKeur Amel, 2008, «Les partis islamistes algériens et la démocratie : vers une professionnalisation politique ? », L'année du Maghreb, n 4, p. 219-238.

CAMAU Michel, GEISSER Vincent, 2003, Le syndrome autoritaire : politique en Tunisie de Bourguiba à Ben Ali, Paris, Presses de Sciences po.

CATUSSE Myriam, (éd.), 2006a, "Les partis politiques dans les pays arabes. Tome 2. Le Maghreb, » Revue des Mondes Musulmans et de la Méditerranée, n 111-112, p. 9-14.

- 2006b, «Ordonner, classer, penser la société : les pays arabes au prisme de l'économie politique », dans La Politique dans le monde arabe, É. PICARD éd., Paris, Armand Colin, p. 215-238.

CHAPRA M. Umer, 2004, « Mawlana Mawdudi’s contribution to Islamic economics », Muslim World, vol. 94, p. 163-180.

CHARIF Maher, 2006, « Réformisme musulman et islam politique : continuité ou rupture ? ", dans P.-J. LuIZARD, Le choc colonial et l’islam, Paris, La Découverte, p. 517-532.

COAKLEY John, 1980, "The Significance of Names: the Evolution of Irish Party Labels ", Études irlandaises, n5, p. 171-182.

CooK Steven A., 2017, False Dawn, Oxford, Oxford University Press.

COURREYe Charlotte, 2016, L'Association des Oulémas Musulmans Algériens et la construction de l'État algérien indépendant_: fondation, héritages, appropriations et antagonismes (1931-1991), Thèse soutenue_en littératures et civilisations, Université Sorbonne Paris GitéINALCO.

DiALMY Abdessamad, 2000, "L’Islamisme marocain : entre révolution et intégration », Archives de sciences sociales des religions, $\mathrm{n}^{\circ} 110$, p. 5-27.

DONEGANI Jean-Marie, 2015, "La sécularisation du croire : pragmatisme et religion », Archives de sciences sociales des religions, $\mathrm{n}^{\circ}$ 1, p. 229-262.

DRIS-AÏT-HAMADOUCHE Louisa, 2009, «Régime et islamistes en Algérie : un échange politique asymétrique ? », Maghreb-Machrek, vol. 2, n² 200, p. 29-44.

Eickelman Dale F., Piscatori James, 1996, Muslim Politics. Princeton, New Jersey, Princeton University Press.

HiBOu Béatrice, 2005, « Tunisie : d'un réformisme à l'autre », Legs colonial et gouvernance contemporaine, $\mathrm{n}^{\circ} 1$, p. 209-263. 
IBN MANZuR Jamal al-Din Abul-Fadl, 2003, Lisân al-'Arab (La langue des Arabes), Beyrouth, Dar Sader.

GREINER Dominique, 2018, « Violence et religions », Revue d'éthique et de théologie morale, $n^{\circ} 4$, p. 61-75.

KEPEL Gilles, 2004, Fitna : Guerre au cœur de l'islam, Paris, Gallimard.

- 2000, Jihad. Expansion et déclin de l'islamisme, Paris, Gallimard.

- 1991, La revanche de Dieu. Chrétiens, juifs et musulmans à la reconquête du monde, Paris, Seuil.

LACROIX Stéphane, 2010, Les islamistes saoudiens, une insurrection manquée, Paris, PUF.

LECOLLE Michelle, 2014, "Dénomination de groupes sociaux : approche sémantique et discursive d'une catégorie de noms propres », SHS Web of Conferences, n 8, p. 2265-2281.

Pastoureau Michel, Simonnet Dominique, 2007, Le petit livre des couleurs, Paris, Seuil.

PAVEAU Marie-Anne, 2008, «Le toponyme, désignateur souple et organisateur mémoriel.

L’exemple du nom de bataille », Mots. Les langages du politique, $\mathrm{n}^{\circ}$ 86, p. 23-35.

REDISSI, Hamadi, 2017, L'Islam incertain : Révolutions et Islam post-autoritaire, Ceres Éditions, Tunis.

RENARD Jean-Bruno, 2011, " Le détournement de sigles. Entre jeu de mots et expression contestataire », Mots. Les langages du politique, p. 29-42.

RougIER Bernard (dir.), 2008, Qu'est-ce que le salafisme ?, Paris, PUF.

Mandaville Peter G., 2003, Transnational Muslim politics: Reimagining the Umma. Routledge.

NiSANYAN Sevan, 2002, Sözlerin Soyagaci [L’arbre généologique des mots], Istanbul, Everest.

Roy Olivier, 2002, L’islam mondialisé, Paris, Points.

- 1992, L'échec de l'islam politique, Paris, Seuil.

SAID Edward, 1984, The World, the Text and the Critic, Londres, Faber and Faber.

SAYYID Bobby S., 1997, A Fundamental Fear: Eurocentrism and the Emergence of Islamism, London, Zed Books.

SENIGUER Haoues, 2013, " Genèse et transformations de l'islamisme marocain à travers les noms. Le cas du Parti de la justice et du développement », Mots. Les langages du politique, $\mathrm{n}^{\circ} 103$, p. 111-120.

SiBLOT Paul, 2001, « De la dénomination à la nomination. Les dynamiques de la signifiance nominale et le propre du nom », Cahiers de praxématique, n 36, p. 189-214.

STEUER Clément, 2013, « S’approprier un nom pour occuper un espace. Le Parti du Centre en Égypte », Mots. Les langages du politique, n 101, p. 113-126. 
STRAUSS Johann, 2008, " Modernisation, nationalisation, désislamisation », Revue des mondes musulmans et de la Méditerranée, no 124, p. 135-159.

TAZAGHART Athmane, 2003, Quelles perspectives pour les partis islamistes modérés ?, Paris, Dossiers du CERI.

TOURNIER Maurice, 2010, Des noms et des gens en République (1879-1914), Paris, L’Harmattan.

ÜNSALDI Levent, 2010, "Les conceptions du développement en Turquie », Revue Tiers Monde, $\mathrm{n}^{\circ} 204$, p. 165-180.

Wolf Anne, 2017, Political Islam in Tunisia: The History of Ennahda, Oxford, Oxford University Press. University Press.

YANKAYA Dilek, 2014, "International Business Forum: une tentative de régionalisation par ta_bourgeoisie islamique turque en "Afro-Eurasie" », Anatoli, nº 5, p. 231-254. 